Since any subgroup of a solvable group is solvable, $G$ cannot be solvable.

\title{
REFERENCES
}

1. R. H. Fox, A quick trip through knot theory, Topology of 3-manifolds, PrenticeHall, Englewood Cliffs, N. J., 1962; pp. 120-167.

2. R. H. Fox and R. H. Crowell, Introduction to knot theory, Ginn, New York, 1963.

3. L. Neuwirth, The algebraic determination of the genus of knots, Amer. J. Math. 82 (1960), 791-798.

Florida State University

\section{THE ENUMERATION OF LABELED TREES BY DEGREES}

\section{BY JOHN RIORDAN}

Communicated by M. Kac, September 28, 1965

1. In [1] Cayley showed that the total number of (free) trees with labeled vertices $v_{1}, \cdots, v_{n}$ is $n^{n-2}$ by exhibiting a correspondence between them and the terms of $\left(v_{1}+\cdots+v_{n}\right)^{n-2} v_{1} v_{2} \cdots v_{n}$. This note shows that the correspondence determines the trees of given degree specification (the degree of a point is the number of lines incident to it; the degree specification is $\left(k_{1}, k_{2}, \ldots\right)$ with $k_{i}$ the number of points of degree $i)$. More precisely, if $T\left(n ; k_{1}, k_{2}, \ldots\right)$ is the number of labeled trees with degree specification $\left(k_{1}, k_{2}, \ldots\right)$ it will be shown that

$$
\begin{aligned}
T_{n}\left(x_{1}, x_{2}, \cdots\right) & =\sum T\left(n ; k_{1}, k_{2}, \cdots\right) x_{1}^{k_{1}} x_{2}^{k_{2}} \cdots \\
& =x_{1}^{n} Y_{n-2}\left(f x_{2} x_{1}^{-1}, \cdots, f x_{n-1} x_{1}^{-1}\right)
\end{aligned}
$$

with $f^{k} \equiv f_{k}=(n)_{k}=n(n-1) \cdots(n-k+1)$, and $Y_{n}$ the Bell multivariable polynomial.

2. In symmetric function notation Cayley's expression is $(1)^{n-2}\left(1^{n}\right)$ on $n$ variables. The multinomial theorem in symmetric function form $[2$, p. 43$]$ is

$(1)^{n}=\sum \frac{n !}{1 !^{k_{1}} \cdots n !^{k_{n}}}\left(1^{k_{1} 2^{k_{2}}} \cdots n^{k_{n}}\right), \quad k_{1}+2 k_{2}+\cdots+n k_{n}=n$.

Hence, on $n$ variables 
(2) $(1)^{n-2}\left(1^{n}\right)=\sum \frac{(n-2) !}{1 !^{k_{1}} \cdots(n-2) !^{k_{n-2}}}\left(1^{n-k^{k_{1}}} 2^{k_{2}} \cdots(n-1)^{k_{n-2}}\right)$

with $k=k_{1}+k_{2}+\cdots+k_{n-2}$. In the symmetric function $\left(1^{n-k} 2^{k_{1}} \ldots\right.$ $\left.(n-1)^{k_{n-2}}\right)$ there are

$$
\frac{n !}{(n-k) ! k_{1} ! \cdots k_{n-2} !}
$$

terms in which $n-k$ variables are of degree 1 , and $k_{i}$ variables are of degree $i+1$. Hence

$$
\text { (1) } \begin{aligned}
T_{n}\left(x_{1}, x_{2}, \cdots\right) & =\sum \frac{(n-2) !(n)_{k} x_{1}^{n}}{k_{1} ! \cdots k_{n-2} !}\left(\frac{x_{2}}{1 ! x_{1}}\right)^{k_{1}} \cdots\left(\frac{x_{n-1}}{(n-2) ! x_{1}}\right)^{k_{n-2}} \\
& =x_{1}^{n} Y_{n-2}\left(f x_{2} x_{1}^{-1}, \cdots, f x_{n-1} x_{1}^{-1}\right), \quad f^{k} \equiv f_{k}=(n)_{k}
\end{aligned}
$$

as stated above; the notation follows [4].

3. Some special cases of (1) are worth noting. First the enumerator by number of points of degree 1 (end points) is $T_{n}(x, 1,1, \ldots)$. But

$$
Y_{n}(x, x, \cdots)=a_{n}(x)=\sum_{k=0}^{n} S(n, k) x^{k}
$$

with $S(n, k)$ the Stirling number of the second kind. Hence

$$
\begin{aligned}
T_{n}(x, 1, \cdots) & =x^{n} Y_{n-2}\left(f x^{-1}, f x^{-1}, \cdots, f x^{-1}\right) \\
& =\sum_{k=0}^{n-2} S(n-2, k) f_{k} x^{n-k} \\
& =\sum_{k=2}^{n} S(n-2, n-k)(n)_{n-k} x^{k}
\end{aligned}
$$

which is given by A. Rényi in [3].

Next the enumerator by number of points of degree 2 is

$$
T_{n}(1, x, 1, \cdots)=Y_{n-2}(f x, f, \cdots, f) .
$$

Since

$$
\begin{array}{r}
\exp u Y\left(f g_{1}, f g_{2}, \cdots\right)=\exp f\left(u g_{1}+u^{2} g_{2} / 2 !+\cdots\right), \\
Y^{k} \equiv Y_{k}, f^{k} \equiv f_{k},
\end{array}
$$

it follows that

$$
\begin{aligned}
\exp u Y(f x, f, \cdots) & =\exp f\left(e^{u}-1-u+u x\right) \\
& =\exp u(b(f)+f x), \quad b^{k}(f) \equiv b_{k}(f),
\end{aligned}
$$


where $b_{n}(s)$ is the associated Stirling number polynomial (enumerating permutations by number of nonunitary ordered cycles) given in $[4$, p. 77]. Hence

$$
\begin{aligned}
T_{n}(1, x, 1, \cdots) & =(b(f)+f x)^{n-2}, \quad f^{k} \equiv f_{k}=(n)_{k} \\
& =\sum_{k=0}^{n-2}\left(\begin{array}{c}
n-2 \\
k
\end{array}\right) x^{k} \sum_{j=0} b_{n-2-k, j}(n)_{k+j}
\end{aligned}
$$

with numbers $b_{n j}$ defined by $b_{n}(s)=\sum b_{n j} s^{j}$ (a short table appears in [4]).

\section{REFERENCES}

1. A. Cayley, $A$ theorem on trees, Quart, J. Math. 23 (1889), 376-378=Collected Papers, Cambridge, 1897, Vol. 13, pp. 26-28.

2. P. A. MacMahon, Combinatory analysis, Vol. I, Cambridge Univ. Press, London, 1915.

3. A. Rényi, Some remarks on the theory of trees, Magyar Tud. Akad. Mat. Kutató Int. Közl. 4 (1959), 73-85. 1958.

4. John Riordan, An introduction to combinatorial analysis, Wiley, New York,

Bell Telephone laboratories, Inc., Murray Hill, New Jersey 